\title{
A Pincer-Shaped Plasma Sheet at Uranus
}

\author{
C. MAX HAMMOND \\ Institute of Geophysics and Planetary Physics, University of California, Los Angeles \\ Department of Earth and Space Sciences, University of California, Los Angeles \\ RAYMOND J. WALKER \\ Institute of Geophysics and Planetary Physics, University of California, Los Angeles \\ MARGARET G. KIVELSON \\ Institute of Geophysics and Planetary Physics, University of California, Los Angeles \\ Department of Earth and Space Sciences, University of California, Los Angeles
}

\begin{abstract}
A model from Voigt et al. (1987) and an MHD simulation from Walker et al. (1989) both show that the curvature of the plasma sheet at Uranus changes as the dipole tilt varies between $38^{\circ}$ and $22^{\circ}$. The models suggest that one of the two partial traversals of the uranian plasma sheet made during the outbound trajectory of Voyager 2 can be explained as an entry into the highly curved plasma sheet that develops when Uranus is near the maximum dipole tilt value of $38^{\circ}$; previously both partial traversals have been explained as anomalous. The spacecraft would have reversed its motion relative to the plasma sheet as the continued rotation diminished the dipole tilt and the retreating plasma sheet uncurled. As the dipole tilt approached its minimum value, spacecraft motion towards the neutral sheet resumed and the traversal of the plasma sheet was completed. Evidence from the PWS plasma wave detector suggests that the spacecraft trajectory skimmed the plasma sheet boundary layer for several hours prior to the partial immersion. The plasma sheet of the Voigt et al. model was not located near the spacecraft during this time interval. On the other hand, the MHD simulation reveals a plasma sheet that is more curved than in the Voigt et al. model; near maximum dipole tilt, the plasma sheet is pincer-shaped. The unusual geometry implies that Voyager 2 remained near the plasma sheet boundary layer during the period suggested by the PWS data. Thus the simulation accounts easily for the first of the plasma sheet encounters previously called anomalous. The second partial immersion remains anomalous, having previously been related to substorm activity, and thus is not discussed here. The stagnation distances of the earth and Uranus at the nose of the magnetopause were used to scale the Walker et al. (1989) simulation of the terrestrial magnetosphere to represent the uranian magnetosphere. The rescaled model was found to predict the radial extent of the magnetotail of Uranus remarkably well. Previous studies which have suggested that the uranian magnetotail is blunter than that of the earth have not compared tail widths at an appropriately scaled distance down the tail.
\end{abstract}

\section{INTRODUCTION}

The unique orientation of the uranian dipole axis and the rotation axis combine to produce a dynamic plasma sheet. As Uranus rotates on its axis, the $8^{\circ}$ angle between the rotation axis and the Sun-Uranus line and the $60^{\circ}$ tilt between the dipole and rotation axes cause the dipole tilt angle to vary between $22^{\circ}$ and $38^{\circ}$ with a period of 17.24 hours, a uranian day [Voigt et al., 1987]. Here the dipole tilt angle is defined as the angle between the dipole axis and $Z_{U S M}$ (refer to the caption of Figure 1 for a definition of USM coordinates). Wu [1984], Walker et al. [1989], and Voigt et al. [1987] have shown that the curvature of the neutral sheet increases with increasing dipole tilt angle. Thus not only does the neutral sheet rotate about the Sun-Uranus line, but also its crosssection changes from a highly curved shape to a less curved, more earth-like shape with a period of one uranian day. Previous studies by Ness et al. [1986] and Behannon et al. [1987] described the average curvature of the uranian plasma

\section{Copyright 1990 by the American Geophysical Union.}

Paper number 90JA00696.

0148-0227/90/90JA-00696\$05.00 sheet by fitting a parabola to the three complete crossings of the neutral sheet. Behannon et al. [1987] using magnetometer data showed that Voyager 2 encountered the plasma sheet five times on its outbound trajectory through the magnetotail. Three of the encounters were full crossings of the plasma sheet in the vicinity of the fitted neutral sheet. Two were partial traversals of the plasma sheet, one somewhat before and one somewhat after the third complete crossing of the neutral sheet. These latter encounters occurred at locations far from the fitted neutral sheet, and are termed partial traversals because the neutral sheet was not crossed. Behannon et al. [1987] have suggested that the two partial traversals result from anomalous motions of the plasma sheet perhaps caused by substorm activity. Mauk et al. [1987] have provided compelling arguments that the immersion following the third crossing was substorm related; it will not be discussed here. This report will attempt to explain the partial traversal of the plasma sheet preceding the third full crossing of the plasma sheet in terms of the slowly varying response of the magnetotail to changing dipole tilt.

Because the Walker et al. [1989] results are based on parameters applicable to the terrestrial magnetosphere the ratio of the Uranus and Earth stand off distances at the nose of the magnetopause was used to scale the results of the Walker 
et al. simulation. Below we show that whether one uses the simulation or fits to observations, the radius of the uranian magnetotail can be predicted from values for the terrestrial magnetosphere if all distances are expressed in terms of the dayside stand off distances. Thus we argue that other aspects of the tail structure can be inferred from the scaled model of the terrestrial magnetosphere.

The particle data used in this study come from the Voyager 2 low energy charged particle (LECP) investigation and were provided by B. Mauk. At Uranus, this experiment was configured to measure electrons of energy $22 \mathrm{keV} \leq \mathrm{E}_{e} \leq 1.2$ $\mathrm{MeV}$ and ions of energy $28 \mathrm{keV} \leq \mathrm{E}_{i} \leq 3.5 \mathrm{MeV}$. During the first two plasma sheet encounters, the instrument used 6 s sector stepping through its eight angular sectors obtaining two angular scans lasting a total of $96 \mathrm{~s}$ followed by a gap of $12 \mathrm{~min}$. After approximately January 25, 19861500 spacecraft event time (SCET), continuous $48 \mathrm{~s}$ sector stepping was used such that a complete angular scan was obtained every $6.4 \mathrm{~min}$. Details of the instrument and its modes have been given by Krimigis et al. [1977, 1981], Armstrong et al. [1981, 1983], Hamilton et al. [1981], and Mauk et al. [1987]. The data from the plasma wave receiver on Voyager 2 used here were obtained from the Planetary Data System. The instrument measures the electric field associated with waves from $10 \mathrm{~Hz}$ to $56 \mathrm{kHz}$. Details of this instrument can be found in Scarf and Gurnett [1977]. The magnetometer data were obtained through the National Space Science Data Center and R. Lepping. The magnetic field experiment onboard Voyager 2 is a three axis fluxgate magnetometer with a sampling rate in all three components of $16.67 \mathrm{~Hz}$ [Ness et al., 1986]. Behannon et al. [1977] contains additional information on this instrument. For this study, the magnetometer data were averaged over $48 \mathrm{~s}$.

\section{DATA}

Figure $1 a$ shows schematically the spacecraft trajectory in the $\mathrm{YZ}_{U S M}$ plane during the interval including the three complete crossings of the plasma sheet (labeled 1,2, and 3 ) and the two partial traversals of the plasma sheet (labeled 3p and 4) identified by Behannon et al. [1987] along with an average plasma sheet shape based on the three complete crossings of the neutral sheet. The spacecraft was also moving tailward during this time. Figure $1 b$ shows the spacecraft trajectory in the $\mathrm{XZ}_{U S M}$ plane. Figure 2 shows that the magnetic field strength is depressed and the particle pressure of the ions measured by the LECP instrument rises at times of crossings and traversals. No plasma sheet signature above the threshold of the Plasma Science experiment (PLS) was found in the low energy ions between $10 \mathrm{eV}$ and $1 \mathrm{keV}$ [Bridge et al., 1986]. The electrons measured by the PLS detector identified only the three complete crossings and the partial immersion labeled 4 [Bridge et al., 1986, Sittler et al., 1987]. Mauk et al. [1987] have shown that the particle energy spectra in the $3 p$ and 3 regions obey similar power laws suggesting similar particle populations. In addition, encounters 1 and $3 p$ are preceded by low frequency wave activity. Based on the spectral form of the waves and their location, Kurth et al. [1989] suggest that the waves may be broadband electrostatic noise. However, since the Voyager instrumentation is incapable of distinguishing between electrostatic and electromagnetic waves for frequencies above $8 \mathrm{~Hz}$ [Kurth et al., 1989] whistler mode waves cannot be ruled out. Figure 2 shows that this

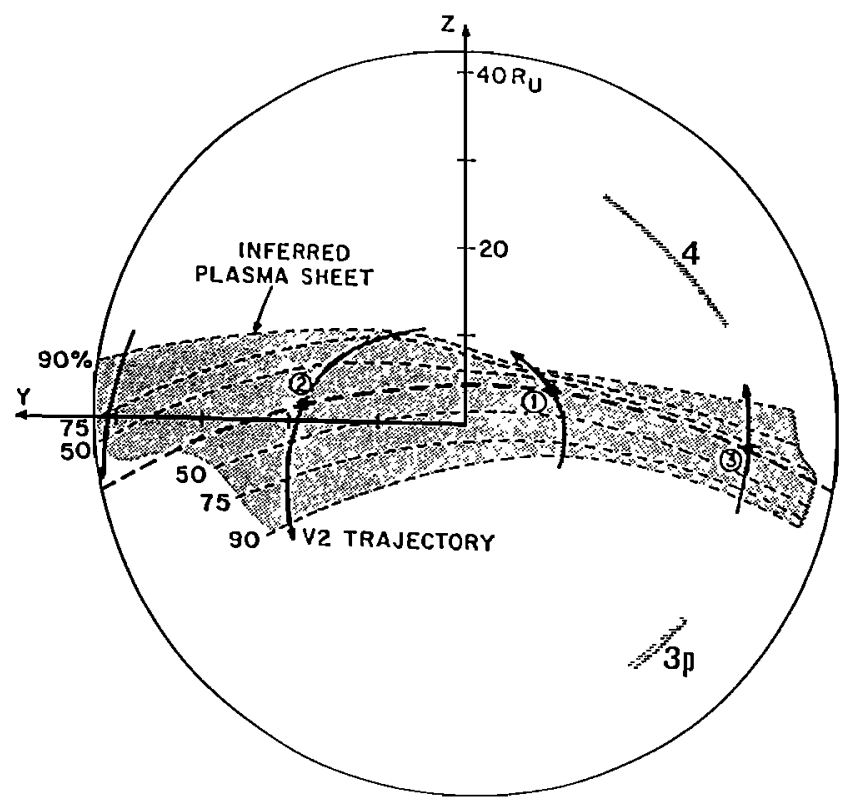

(a)

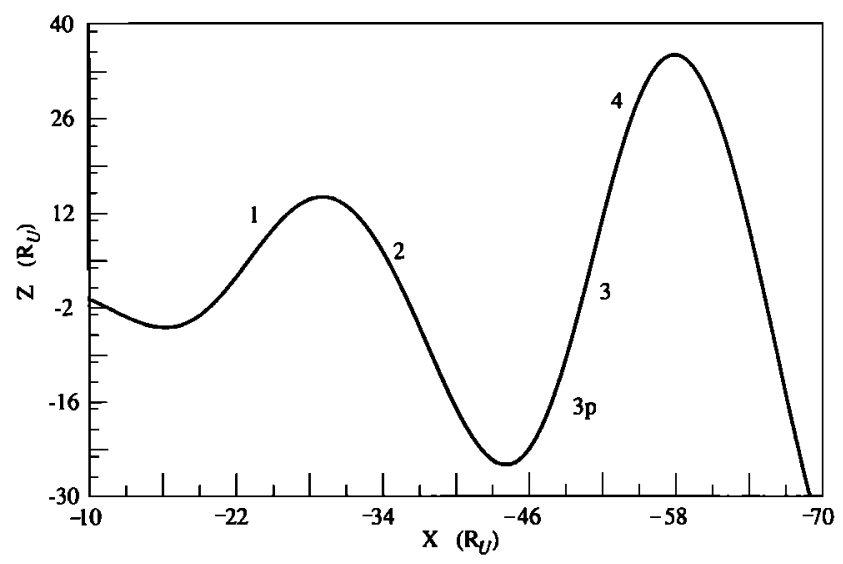

(b)

Fig. 1. (a) Cross section of the plasma sheet in USM coordinates (planet centered cartesian coordinates: $X$ positive toward the sun, $X Z$ plane contains the dipole, and $\mathrm{Y}$ completes the right handed coordinate system) inferred from decreases of magnetic field magnitude. The shaded region represents the plasma sheet while the heavy dashed line shows the shape of the average neutral sheet. Lighter dashed lines show positions at which the field magnitudes were 50,75, and $90 \%$ of the lobe magnetic field intensity. Continuous black and gray lines represent portions of Voyager's trajectory. Numbered segments identify plasma sheet encounters and are centered at times indicated and numbered in Figure 2. Adapted from Behannon et al. [1987]. (b) Trajectory of Voyager 2 in the $X Z_{U S M}$ plane. Plasma sheet encounters are identified as in Figure $1 a$.

wave activity is not present prior to the second plasma sheet crossing when the spacecraft was in the northern lobe.

Broadband electrostatic noise would be consistent with a spacecraft location within a plasma sheet boundary layer in the southern (night side) lobe prior to the entrance into the plasma sheet. However, the absence of wave activity in the northern lobe is hard to reconcile with the interpretation of the wave data as noise associated with an encounter of the plasma sheet boundary layer. A possible explanation of the north-south asymmetry may be that the northern (southern) lobe field lines are always connected to the day side (night side). It is 


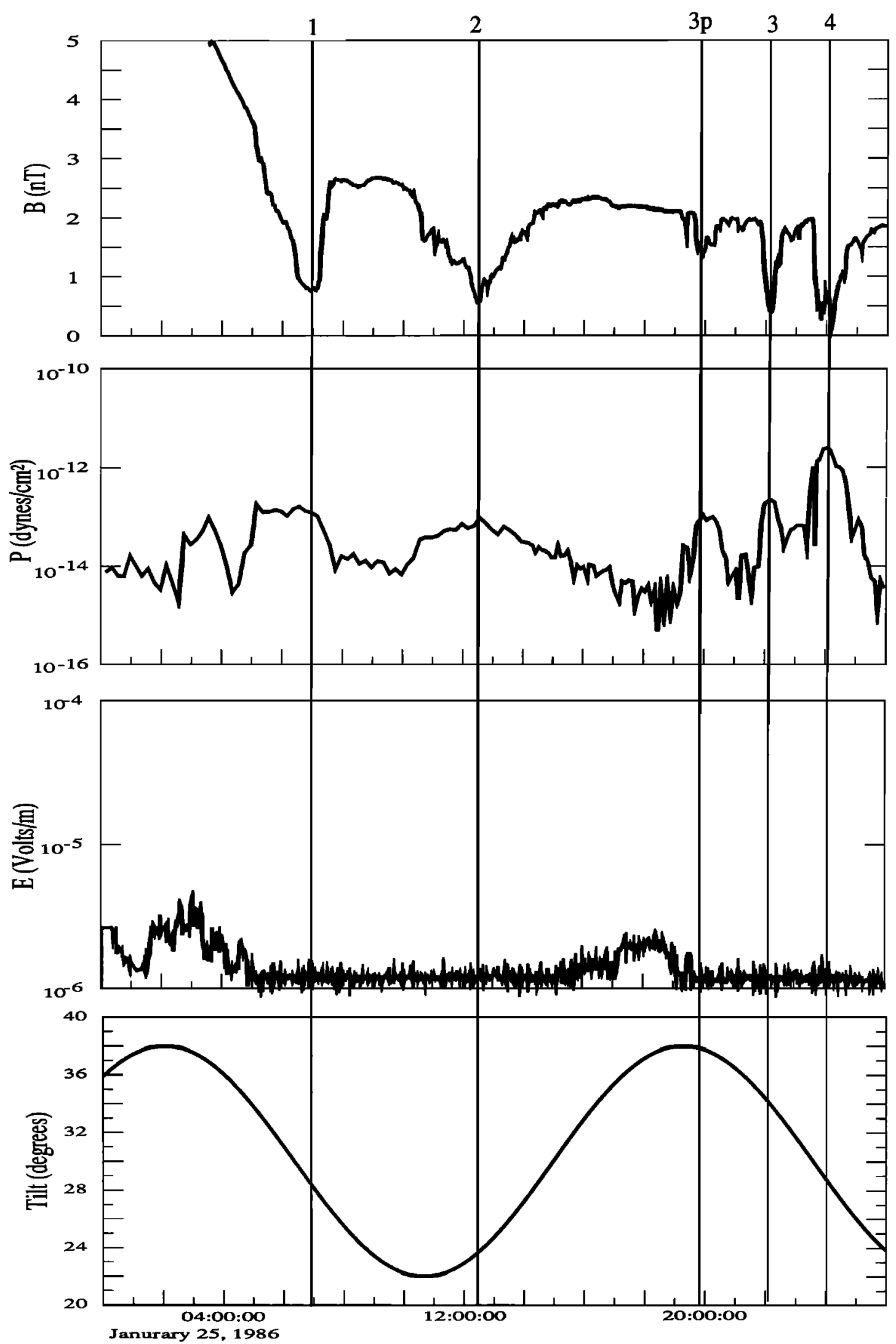

Fig 2. Magnetic field strength, pressure of $>28 \mathrm{keV}$ ions from the LECP investigation, electric field from the $31.1 \mathrm{~Hz}$ PWS channel, and dipole tilt angle versus spacecraft event time. Plasma sheet encounters are numbered to correspond to the labels in Figure 1. 
possible the associated asymmetry in boundary conditions at the ionospheric feet of the fluxtubes may cause an asymmetry in particle distributions creating distributions in the southern lobe that are more conducive to instabilities which drive the plasma waves [Kurth et al., 1989]. We accept this explanation of the absence of wave activity in the northem lobe. Having done so, it is reasonable to associate the observed broadband electrostatic noise with a close approach to the southern lobe boundary of the plasma sheet.

Clearly the immersions into the plasma sheet labeled 3p and 4 are not consistent with the average shape of the plasma sheet presented by Behannon et al. [1987] and shown in Figure 1a. As mentioned previously, immersion 4, which will not be treated here, appears to be substorm related and thus not representative of the quasi-static plasma sheet structure. As Figure 2 shows, the 3p traversal occurred at a time when the dipole tilt angle was near maximum and decreasing. A tempting interpretation then is that this immersion was not an anomalous event but instead resulted from a large scale plasma sheet structure unique to Uranus, the plasma sheet being highly curved at a time of the extreme $38^{\circ}$ dipole tilt angle. The fact that the dipole tilt angle decreased steadily for eight hours after the $3 p$ event could explain why the spacecraft left the plasma sheet and did not pass through the neutral sheet until crossing 3; the decreasing dipole tilt would diminish the curvature of the plasma sheet and pull the plasma sheet away from the spacecraft.

Figure 3 shows the plasma sheet configuration for dipole tilt angles of $22^{\circ}$ and $38^{\circ}$ given by Voigt et al. [1987] overlaid by hourly dots representing the trajectory of Voyager 2 in uranian solar magnetospheric (USM) coordinates. The increasing curvature of the plasma sheet with increasing dipole tilt is evident. The figure shows that the spacecraft approached the model plasma sheet at the time of the anomalous $3 p$ crossing. The hash marks along the trajectory in the figure identify the interval immediately before the $3 \mathrm{p}$ immersion during which low frequency wave activity was observed. The spacecraft appears to have approached the plasma sheet approximately along the local normal. Although the Voigt et al. model does not explicitly show a plasma sheet boundary layer, the spacecraft trajectory does not appear to have been near a plausible location of the plasma sheet boundary layer for this model during the period of plasma wave activity. Indeed, for the nearly normal approach, one might anticipate that the spacecraft would spend a short residence time in the plasma sheet boundary layer rather than the approximately three hours suggested by our interpretation of the PWS measurements.

A limitation of the Voigt et al. model is that it uses a fixed magnetopause. $W u$ [1984] and Walker et al. [1989] point out that a similar model for the Earth with a fixed magnetopause [Voigt, 1984] underestimates the observed curvature of the terrestrial plasma sheet. With a fixed magnetopause the only way to maintain equal magnetic flux in each lobe as the dipole tilt changes is through curvature of the plasma sheet. However, it seems likely that conservation of magnetic flux in the lobes is partially produced by distortion of the magnetopause surface. In MHD simulations, the magnetopause is not a prescribed boundary but is allowed to develop as a simulated solar wind flows over a dipole; the shape of the magnetopause distorts as a function of dipole tilt and this presumably more closely resembles reality. As the magnetopause shape changes, the shape of the plasma sheet also changes. Figure 4 shows cross sections of the magnetotail and the plasma sheet shape for
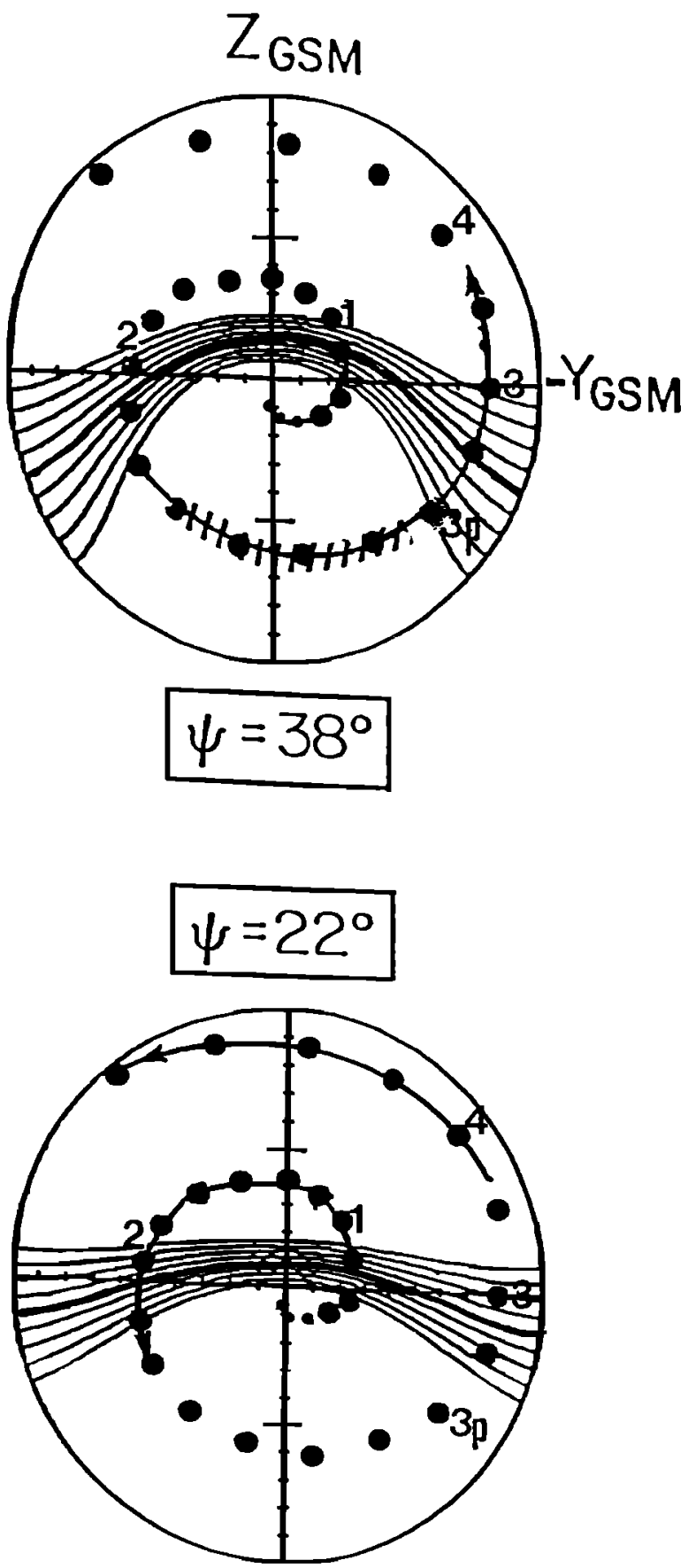

Fig 3. Current streamlines showing the plasma sheet shape for dipole tilt angles of $22^{\circ}$ and $38^{\circ}$ from the Voigt et al. [1987] model. The heavy line indicates the neutral sheet. Voyager's trajectory is represented by hourly dots. Solid curves link the dots for periods in the trajectory where the actual dipole tilt angle was closest to the dipole tilt angle shown in the figure. Numbered labels as in Figure 2 identify the encounters with the plasma sheet. Hash marks identify the interval during which plasma wave activity was significant immediately before the $3 \mathrm{p}$ event. Figure adapted from Voigt et al. [1987].

dipole tilts of $20^{\circ}, 30^{\circ}$, and $38^{\circ}$ from MHD simulations by Walker et al. [1989]. The distortion of the magnetopause surface and changing curvature of the plasma sheet can be seen. Note that at Uranus the minimum dipole tilt angle is $22^{\circ}$.

The lengthy computer time needed for the runs used for the Walker et al. paper precluded a fully time dependent calculation. Instead, their results were produced separately 

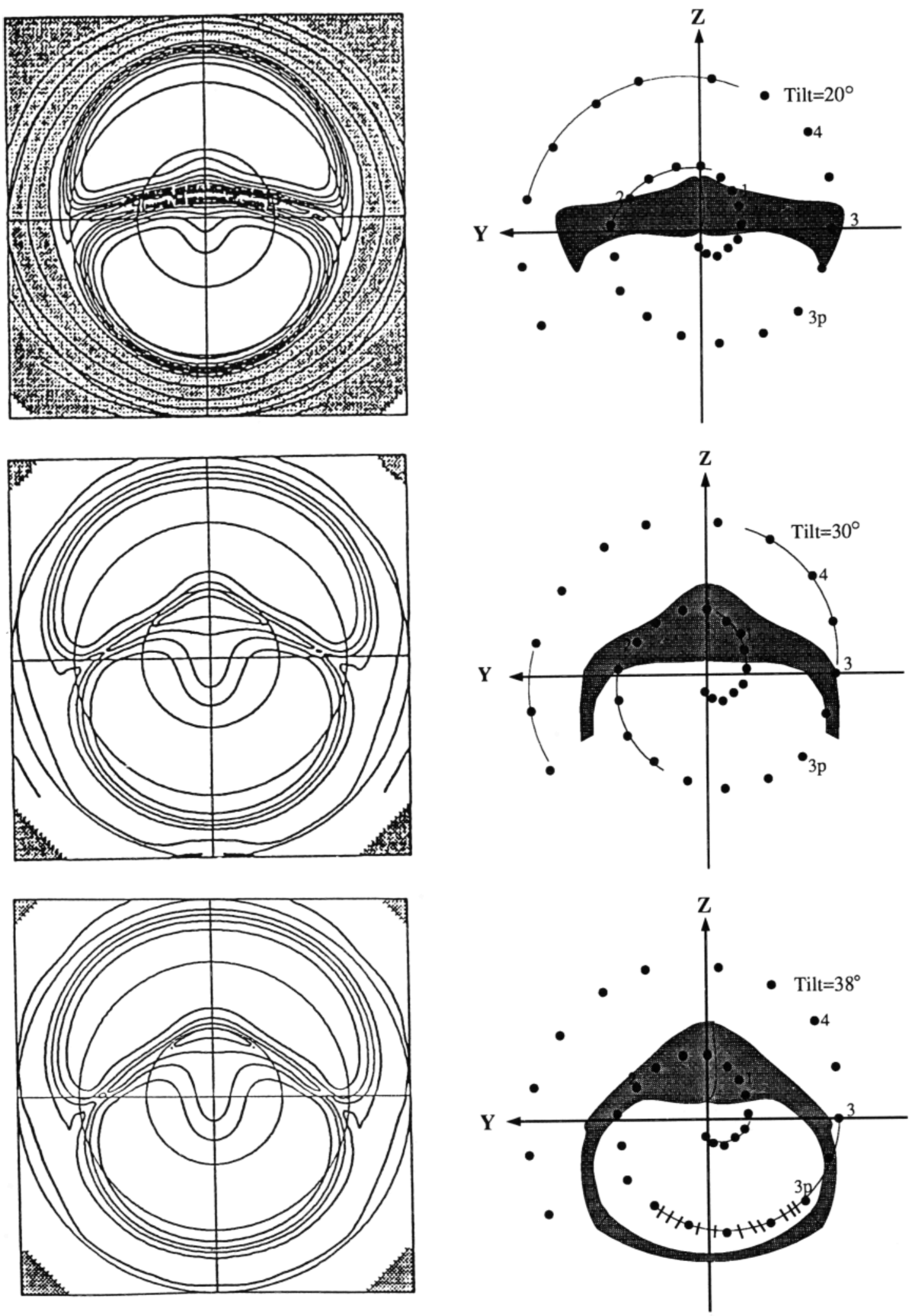

\section{Pressure Contours}

Fig. 4. Pressure contours from the MHD simulations of Walker et al. [1989] for dipole tilt angles of $20^{\circ}, 30^{\circ}$, and $38^{\circ}$ (top to bottom). The boxes are cut at constant $\mathrm{X}_{U S M}$ across the magnetotail and the width of each box is $103 \mathrm{R}_{U}$ for the scaling adopted in this paper. The $20^{\circ}$ case corresponds to a down tail distance of $31 R_{U}$ while the $30^{\circ}$ and $38^{\circ}$ cases correspond to down tail distances of $61 \mathbf{R}_{U}$. A sketch of the plasma sheet shape is shown beside each contour plot. The plasma sheet boundary for each case was determined in the manner described in the text. Dots show the spacecraft position at one hour intervals. Solid curves link the dots for periods in the trajectory where the dipole tilt angle shown in the figure was closest to the actual dipole tilt angle. Numbered labels identify the encounters with the plasma sheet as in Figure 1. Hash marks identify the interval during which plasma wave activity was significant immediately before the $3 p$ event. 
for each dipole tilt angle. As a result, the twist in the tail observed by Behannon et al. [1987] due to the effect of a finite Alfven speed in propagating the changing tail structure down the tail has been ignored in the Walker et al. simulation as in the Voigt et al. model.

The simulations were run with parameters applicable to the Earth's magnetosphere. To apply the results to Uranus we have scaled distances by the ratio, $\rho$, of the Uranus and Earth standoff distances at the nose of the magnetopause:

$$
\rho=18 \mathbf{R}_{U} / 10.7 \mathbf{R}_{E}=1.68 \mathbf{R}_{U} / \mathbf{R}_{E}
$$

Behannon et al. [1987] found that the normalized tail radii of Earth, Jupiter, Saturn, and Uranus, scaled in this manner are approximately equal.

It is not intuitively obvious why a scaling by stagnation distances yields a quantitative model for the uranian tail. Far down the tail, the tail current is responsible for confining the field. The current in the tail current sheet is governed by the thermal plus magnetic pressure of the solar wind whereas the stagnation distance varies with the dynamic pressure of the solar wind. The thermal and magnetic pressure of the solar wind decrease with distance from the sun more rapidly than the dynamic pressure. One might expect a scaling by stagnation distances to considerably underestimate distances which are governed by the thermal and magnetic pressures of the solar wind. However, the scaled simulation of Walker et al. predicts a tail radius of $42 R_{U}$ at the point where Voyager 2 left the magnetopause. This is in good agreement with the observed value of $42.9 \mathrm{R}_{U}$ [Bridge et al., 1986]. The success of this scaling factor may be because the radius of the magnetotail depends not only on the lobe field strength but also on the size of the polar cap, thus depending on both the tail currents and the Chapman-Ferraro currents. It is not immediately evident which are more important. At some distance down the tail, determined by the thermal pressure of the solar wind, the Chapman-Ferraro current ends and the tail current begins. Conservation of the Chapman-Ferraro current can be used to determine the tail radius at this point in terms of the distance of the dayside neutral point from the planetsun line [Unti and Atkinson, 1968]. Further down tail, the flaring angle of the tail still depends strongly on the dynamic pressure as long as it exceeds the other solar wind pressure forces. Thus the flaring angle must be similar for the earth and Uranus. It is then easy to understand why magnetotails scaled by the stagnation distances would be similar for earth and Uranus. Because of tail flaring one must be careful to compare tail radii at equivalent down tail scaled distances. Behannon et al. [1987], using rough estimates, found that the scaled tail radii were approximately equal for the earth and Uranus, citing values of 1.8 for the earth and 2.2 for Uranus. A more precise value for Uranus based on PLS data is 2.4 [Bridge et al., 1986]. The terrestrial value of 1.8 is valid near the terminator. As the tail radius continues to increase with distance down the tail, the scaled radius increases down the tail. An expression for the radius, $S$, of the terrestrial tail as a function of distance down the tail has been developed by Howe and Binsack [1972] using data from Explorer 33. In units of stagnation distance, the expression becomes

$$
S(x)=2.23 \arctan \sqrt{\frac{0.93-x}{1.46}}
$$

Applied to Uranus, this expression predicts that at $\mathrm{x}=-3.8$ stagnation units down the tail of Uranus, which is where the Voyager spacecraft crossed the magnetopause, the tail radius is $S=2.4$, or $43 R_{U}$. This result is in agreement with the simulation result and supports our use of the scaled terrestrial simulation for Uranus.

For the scaling described, the width of each box shown in Figure 4 corresponds to $100.8 R_{U}$. The cross section shown for the case of a dipole tilt angle of $20^{\circ}$ is a cut at a down tail distance of $31 R_{U}$ to correspond approximately to the 35 $\mathbf{R}_{\boldsymbol{U}}$ down tail position of Voyager 2 at the time of the second neutral sheet crossing which occurred when the dipole tilt was near $20^{\circ}$. The results shown for the $30^{\circ}$ and $38^{\circ}$ cases are for a down tail distance of $61 \mathrm{R}_{U}$ to approximately correspond to the down tail distance of Voyager 2 during the $3 p$ and 3 immersions into the plasma sheet.

In the pressure contour plots shown it is difficult to identify the boundary between the plasma sheet and the magnetosheath. However, in the analogous simulation for dipole tilt angles greater than $38^{\circ}$, the plasma sheet is detached along the bottom edge of the magnetopause at a dipole tilt angle of $45^{\circ}$ and it curls up with increasing tilt, forming a cylindrical plasma sheet for a dipole tilt angle of $90^{\circ}$ [Ogino et al., 1989]. Figure 5 shows the neutral sheet position in the noon-midnight meridian plane of the simulated magnetosphere as a function of dipole tilt angle. Note that at $45^{\circ}$ the position of the neutral sheet is double valued indicating that the neutral sheet has detached along the bottom to form a distorted cylindrical plasma sheet. Figure 5 indicates that the plasma sheet for a dipole tilt angle of $38^{\circ}$ would adopt a distorted cylindrical shape.

Using Figure 5 as a guide to the interpretation of color plots of the pressure for a series of dipole tilt angles one can identify the boundary between the plasma sheet and the magnetosheath. This method was used to determine the plasma sheet shapes sketched beside each contour plot shown in Figure 4 . The dynamic nature of the plasma sheet is evident from Figure 4. The plasma sheet is only slightly curved near the minimum dipole tilt angle of $22^{\circ}$. As the tilt angle increases the plasma sheet becomes more curved until it is almost pincer-shaped, resembling the lower portion of a pair of ice tongs. Hourly dots representing the spacecraft's trajectory have been overlaid on each of the three plots. Dots are connected with smooth curves along that part of the trajectory for which the modeled dipole tilt angle of the figure is closest to the actual dipole

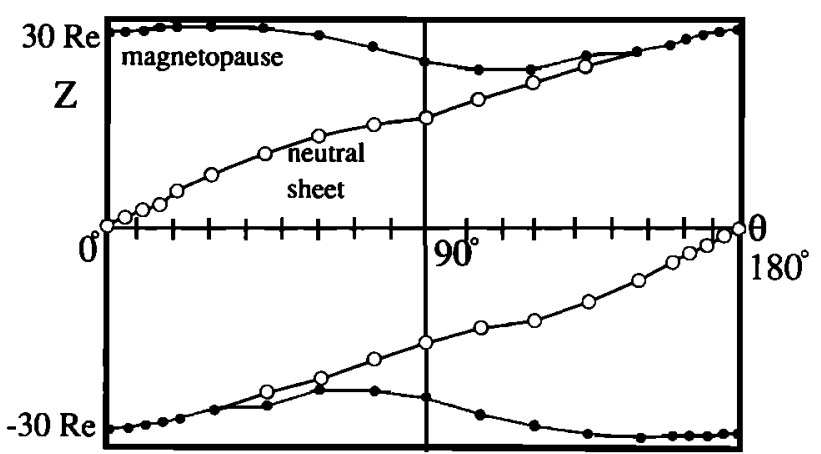

Fig. 5. Magnetopause position (solid circles) and neutral sheet position (open circles) versus dipole tilt angle from a simulation from Ogino et al. [1989] analogous to the Walker et al. [1989] simulation presented in the text. Note that the neutral sheet position becomes double valued indicating the plasma sheet has become roughly cylindrical. Figure adapted from Figure 11 of Ogino et al. [1989]. 
tilt angle. At the time of the first plasma sheet crossing the dipole tilt angle was decreasing. By the time of the second crossing the plasma sheet was only slightly curved but the curvature was increasing. The "pincers" were closing after the second plasma sheet crossing and continued to close until just before the 3p plasma sheet encounter. In Figure 4, the region of plasma wave activity before the 3 p encounter has been identified with hash marks. The figure shows that prior to the $3 p$ immersion, the spacecraft moved almost tangentially to the plasma sheet and could have been close enough to the plasma sheet to have been in or very close to the plasma sheet boundary layer. This would account for the presence of plasma waves. At the time of the $3 p$ partial immersion in the plasma sheet, the dipole tilt angle was near its maximum of $38^{\circ}$ and decreasing (Figure 2). As seen in the lower panel of Figure 4 the spacecraft would have just been entering the plasma sheet at this time (1945 SCET) which is consistent with the data. As the dipole tilt began to decrease, the plasma sheet began to flatten as shown in the middle panel of Figure 4, causing the spacecraft to neutral sheet distance to increase as the pincers pulled away from the spacecraft. The distance between the neutral sheet and spacecraft then decreased as Voyager overtook the retreating plasma sheet at crossing 3 as shown in the middle panel of Figure 4 . The third neutral sheet crossing occurred at 2209 SCET. The absence of more low frequency wave activity and the fact that the ion pressure did not fall back to the level observed before the $3 p$ immersion indicate that the spacecraft probably did not completely leave the plasma sheet between the 3p partial traversal and the third complete crossing as the pincers of the plasma sheet pulled away from the spacecraft, consistent with the diagrams of Figure 4. Both the MHD simulations and the data show that the spacecraft moved into the northem lobe after approximately 2230. Mauk et al. [1987] have shown that the plasma sheet then probably expanded over the spacecraft as a result of substorm activity. This then accounts for the final anomalous immersion in the plasma sheet labeled 4.

\section{DISCUSSION}

Both the Voigt et al. [1987] model and the Walker et al. [1989] MHD simulations of the plasma sheet predict that Voyager 2 should have entered the plasma sheet at the time of the $3 p$ encounter. Plasma wave activity was high prior to this encounter. As Kurth et al. [1989] point out, it is impossible to tell if the observed wave activity was electrostatic or electromagnetic in nature because the magnetometer onboard Voyager 2 cannot measure magnetic fluctuations above approximately $8 \mathrm{~Hz}$. Thus the whistler mode cannot be ruled out for the plasma wave activity prior to the 3p encounter, but Kurth et al. [1989] favor the broadband electrostatic interpretation. Broadband electrostatic waves are frequently observed in the plasma sheet boundary layer at Earth [Gurnett et al., 1976] and Jupiter [Barbosa et al., 1981]. If the PWS signals were broadband electrostatic waves, then that would seem to indicate that Voyager was in or near to the plasma sheet boundary layer prior to the $3 p$ immersion. For a plasma sheet of the shape given by Voigt et al. [1987] in Figure 3, the trajectory remained far from the region of a nominal plasma sheet boundary layer during the pertinent interval. The spacecraft was heading almost perpendicularly into the plasma sheet. In contrast, the MHD simulation shows that Voyager 2 skirted the edge of the plasma sheet during the interval of plasma wave activity and it is reasonable to suppose that the spacecraft may have been in the plasma sheet boundary layer.

An alternate explanation of the wave activity is that these waves were associated with the magnetopause boundary layer [Kurth et al., 1989]. Figure 3 shows that the Voigt et al. model is also inconsistent with this interpretation because the magnetopause boundary layer was approximately $15 \mathrm{R}_{U}$ from the region of plasma wave activity.

\section{CONCLUSION}

Plasma sheet expansion or anomalous torquing of the plasma sheet need not be invoked to explain the $3 \mathrm{p}$ immersion of the Voyager 2 spacecraft in the uranian plasma sheet. Magnetic field and LECP particle data show the characteristic diamagnetic depression and particle pressure enhancement associated with plasma sheet encounters. Unlike plasma sheet immersion 4 in which particle spectra became harder than previous spectra [Mauk et al., 1987], the 3p immersion has no obvious spectral anomalies that would suggest a relation to a substorm. Both the Voigt et al. [1987] model and the MHD simulations show that the curvature of the plasma sheet can account for the $3 p$ immersion, but only the MHD simulation provides a plausible explanation of the plasma wave data in the interval prior to and including the immersion.

The interpretation requires that the scaled MHD simulations correctly represent the uranian magnetosphere. We have shown that the scaling produces remarkable agreement in the tail widths observed for the earth and Uranus, provided that the tail radius is measured at identically scaled distances down the tail. Thus it seems that the stagnation distance is an appropriate scaling factor to use when comparing the magnetospheric cavities of the earth and Uranus. When distances are scaled in this manner, there is no need to argue that the magnetosphere of Uranus is blunter than that of the earth as suggested by Bridge et al. [1986]. Furthermore, the success of this scaling implies that within the tail volume traversed by Voyager 2 the Chapman-Ferraro currents control the magnetotail radius.

In summary, we have shown that an MHD simulation of the terrestrial magnetosphere, rescaled to represent the uranian magnetotail, can account for some puzzling plasma properties. We have been able to explain the $3 p$ immersion, previously regarded as being anomalous, in terms of the possibility of extreme departures from average plasma sheet shapes in the uranian magnetosphere. The unusual orientation of the uranian dipole and rotation axes produce a dynamically curved plasma sheet which is an unusual feature of the uranian magnetosphere.

Acknowledgments. We thank R. Lepping and the National Space Science Data Center for supplying the magnetometer data, B. Mauk for providing LECP data, and W. Kurth and the Planetary Data System for supplying the plasma wave data We especially thank T. Ogino for his help in interpreting the simulations. Computing resources were supplied by both the San Diego Supercomputing Center and the Research Institute of Atmospherics of Nagoya University. This work was supported by NASA grant NAGW-1179. Institute of Geophysics and Space Physics Publication 3238.

The Editor thanks K.-H. Glassmeier and R. S. Selesnick for their assistance in evaluating this paper.

\section{REFERENCES}

Armstrong, T. P., M. T. Paonessa, S. I. Brandon, S. M. Krimigis, and L. J. Lanzerotti, Low-energy charge 


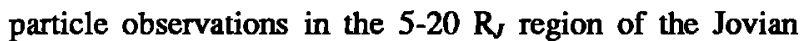
magnetosphere, J. Geophys. Res., 86, 8343, 1981.

Armstrong, T. P., M. T. Paonessa, E. V. Bell II, and S. M. Krimigis, Voyager observations of Saturnian ion and electron phase space densities, J. Geophys. Res., 88, 8893, 1983.

Barbosa, D. D., F. L. Scarf, W. S. Kurth, and D. A. Gurnett, Broadband electrostatic noise and field-aligned currents in Jupiter's middle magnetosphere, J. Geophys. Res., 86, 8357, 1981.

Behannon, K. W., M. H. Acuna, L. F. Burlaga, R. P., Lepping, and N. F. Ness, Magnetic Field Experiment For Voyager 1 and 2, Space Sci. Rev., 21, 235, 1977.

Behannon, K. W., R. P. Lepping, E. C. Sittler, Jr., N. F. Ness, B. H. Mauk, S. M. Krimigis, and R. L. McNutt, Jr., The magnetotail of Uranus, J. Geophys. Res., 92, 15354, 1987.

Bridge, H. S., et al., Plasma observations near Uranus: Initial results from Voyager 2, Science, 233, 89, 1986.

Gurnett, D. A., L. A. Frank, and R. P. Lepping, Plasma waves in the distant magnetotail, J. Geophys. Res., 81, 6059, 1976.

Hamilton, D. C., G. Gloeckler, S. M. Krimigis, and L. J. Lanzerotti, Composition of nonthermal ions in the Jovian magnetosphere, J. Geophys. Res., 86, 8301, 1981.

Howe, H. C. and J. H. Binsack, Explorer 33 and 35 Plasma Observations of Magnetosheath Flow, J. Geophys. Res., 77, 3334, 1972.

Krimigis, S. M., T. P. Armstrong, W. I. Axford, C. 0. Bostrom, C. Y. Fan, G. Gloeckler, and L. J. Lanzerotti, The low energy charged particle (LECP) experiment on the Voyager spacecraft, Space Sci. Rev., 21, 329, 1977.

Krimigis, S. M., J. F. Carbary, E. P. Keath, C. O. Bostrom, W. I. Axford, G. Gloeckler, L. J. Lanzerotti, and T. P. Armstrong, Characteristics of hot plasma in the Jovian magnetosphere: Results from the Voyager spacecraft, $J$. Geophys. Res., 86, 8227, 1981.

Kurth, W. S., D. A. Gurnett, F. L. Scarf, and B. H. Mauk, Plasma waves in the magnetotail of Uranus, J. Geophys. Res., 94, 3505, 1989.
Mauk, B. H., S. M. Krimigis, E. P. Keath, A. F. Cheng, T. P. Armstrong, L. J. Lanzerotti, G. Gloeckler, and D. C. Hamilton, The hot plasma and radiation environment of the uranian magnetosphere, J. Geophys. Res., 92, 15283, 1987.

Ness, N. F., M. H. Acuna, K. W. Behannon, L. F. Burlaga, J. E. P. Connemey, R. P. Lepping, and F. M. Neubauer, Magnetic fields at Uranus, Science, 233, 85, 1986.

Ogino, T., R. J. Walker, and M. Ashour-Abdalla, The effects of dipole tilt on magnetotail structure and dynamics, Proc. Res. Inst. Atmos. Nagoya Univ. 36, 29, 1989.

Scarf, F. L., and D. A. Gurnett, A plasma wave investigation for the Voyager mission, Space Sci. Rev., 21, 289, 1977.

Sittler, E. C. Jr., K. W. Ogilvie, and R. S. Selesnick, Survey of electrons in the Uranian magnetosphere: Voyager 2 observations, J. Geophys. Res., 92, 15263, 1987.

Unti, T. and G. Atkinson, Two-dimensional Chapman-Ferraro problem with neutral sheet 1 . The boundary, J. Geophys. Res., 73, 7319, 1968.

Voigt, G. H., The shape and position of the plasma sheet in the earth's magnetotail, J. Geophys. Res., 89, 2169, 1984.

Voigt, G. H., K. W. Behannon, and N. F. Ness, Magnetic field and current structures in the magnetosphere of Uranus, $J$. Geophys. Res., 92, 15337, 1987.

Walker, R. J., T. Ogino, and M. Ashour-Abdalla, Simulating the magnetosphere: The structure of the magnetotail, in Solar System Plasma Physics, Geophys. Monogr. Ser., vol. 54, edited by J. H. Waite Jr. et al., p. 61, AGU Washington, D. C., 1989.

Wu, C. C., The effects of dipole tilt on the structure of the magnetosphere, J. Geophys. Res., 89, 11048, 1984.

C. M. Hammond, M. G. Kivelson, and R. J. Walker, Institute of Geophysics and Planetary Physics, Slichter Hall, University of California, Los Angeles, CA 90024-1567.

(Recieved August 31, 1989;

revised March 1, 1990;

accepted March 16, 1990.) 